\title{
Current Options and Practices in Long-Term Ventricular Assist Devices
}

\author{
Jacob R. Miller • Christopher P. Lawrance • \\ Scott C. Silvestry
}

Published online: 27 March 2014

(C) Springer Science + Business Media New York 2014

\begin{abstract}
End-stage heart failure poses a significant problem for patients and a burden on the healthcare system. With a lack of available hearts for transplant, mechanical circulatory support has emerged as a viable treatment option. The first generation ventricular assist device (VAD) showed superior outcomes to optimal medical management. Now with second and third generation continuous-flow VADs available, outcomes have continued to improve. Patients who undergo VAD implantation have superior survival and increased quality of life; however, they also have a significant risk of adverse events. These adverse events are decreasing as newer devices are developed, surgical experience increases, postoperative care improves and patient selection trends toward less critically ill patients. Future progress of the VAD will continue to improve survival and decrease adverse events.
\end{abstract}

Keywords Ventricular assist device .

Mechanical circulatory support - Destination therapy ·

Bridge to transplant $\cdot$ End-stage heart failure

\section{Introduction: Heart Failure}

Heart failure represents a significant challenge to the current state of health care. Despite advances in medical

This article is part of the Topical Collection on Artificial Organ CT Surgery.

J. R. Miller · C. P. Lawrance · S. C. Silvestry $(\square)$

Division of Cardiothoracic Surgery, Department of Surgery,

Barnes-Jewish Hospital, Washington University School of

Medicine, CB 8234, 660 South Euclid Avenue, 63110 St. Louis, MO, USA

e-mail: silvestrys@wudosis.wustl.edu therapy, heart failure is still the primary diagnosis in $>1$ million hospitalizations with a 1 -month readmission rate approaching $25 \%[1,2]$. Heart failure also poses a significant financial burden. Heart failure related costs have already exceeded $\$ 30$ billion in the US for 2013 with a mean heart failure-related hospitalization costing $\$ 23,077$ per patient [3]. Progression to the end stages of heart failure is marked by severe activity intolerance, frequent hospitalizations, need for IV inotropic support and immanent death. The best available treatment option is a heart transplant with a 10-year survival of $55 \%$; unfortunately with only 2,200 donor hearts available per year, this treatment option is realized by few patients [4]. A prolonged time spent on the heart transplant waiting list is associated with significant morbidity and mortality, and it was for this short-coming that mechanical circulatory support (MCS) was developed and has subsequently evolved into an excellent option for many patients.

Heart failure is traditionally classified into NYHA classifications based on the patient's functional status. However, in order to classify the patients more precisely, seven Interagency Registry for Mechanically Assisted Circulatory Support (INTERMACS) patient profiles with three modifiers were created (Table 1). This information was meant to aid in the assessment of patient outcomes with mechanical circulatory support as well as risk stratify potential patients for MCS [5].

\section{Mechanical Circulatory Support}

Mechanical circulatory support encompasses multiple therapeutic options for treating the most advanced stages of heart failure in either the acute or chronic setting. Shortterm devices include the intra-aortic balloon pump, Impella 
Table 1 The 7 INTERMACS profile

\begin{tabular}{|c|c|c|}
\hline Profile & Title & Description \\
\hline 1 & $\begin{array}{l}\text { Critical } \\
\text { cardiogenic } \\
\text { shock }\end{array}$ & $\begin{array}{l}\text { Life-threatening hypotension refractory to } \\
\text { IV inotropes. "crash and burn" }\end{array}$ \\
\hline 2 & $\begin{array}{l}\text { Progressive } \\
\text { decline }\end{array}$ & $\begin{array}{l}\text { IV inotropes required with worsening } \\
\text { end-organ function. "sliding on } \\
\text { inotropes" }\end{array}$ \\
\hline 3 & $\begin{array}{l}\text { Inotrope } \\
\text { dependent }\end{array}$ & $\begin{array}{l}\text { Stable blood pressure and end-organ } \\
\text { function but failure to wean from IV } \\
\text { inotropes. "dependent stability" }\end{array}$ \\
\hline 4 & $\begin{array}{l}\text { Resting } \\
\text { symptoms }\end{array}$ & $\begin{array}{l}\text { Daily symptoms of congestion at rest or } \\
\text { with ADLs. High doses of diuretics }\end{array}$ \\
\hline 5 & $\begin{array}{l}\text { Exertion } \\
\text { intolerant }\end{array}$ & $\begin{array}{l}\text { Unable to engage in any activity above } \\
\text { ADLs }\end{array}$ \\
\hline 6 & $\begin{array}{r}\text { Exertion } \\
\text { limited }\end{array}$ & $\begin{array}{l}\text { Can participate in minor activities but } \\
\text { quickly fatigues. "walking wounded" }\end{array}$ \\
\hline 7 & $\begin{array}{l}\text { Advanced } \\
\text { NYHA III }\end{array}$ & $\begin{array}{l}\text { Comfortable with meaningful activity, } \\
\text { limited to mild exertion }\end{array}$ \\
\hline
\end{tabular}

Three modifiers: TCS temporary circulatory support, $A$ arrhythmia, $F F$ frequent flyer. Adapted from Stevenson et al. [5]

(Abiomed, Danvers, MA), Tandem Heart Percutaneous Ventricular Assist Device (Cardiac Assist, Inc, Pittsburgh, PA), CentriMag (Levitronix, Waltham, MA) and extracorporeal membrane oxygenation. However, only implantable ventricular assist devices (VAD) are suitable for durable long-term support. Implantation of a VAD has been shown to increase survival while waiting on a heart transplant list and represents an excellent surgical option for patients who are not candidates for a transplant.

\section{Indications}

VADs were developed initially as a "bridge to transplant" (BTT) therapy to enable critically ill patients to survive to cardiac transplant. After clinical studies demonstrated effectiveness in the transplant population, VAD therapy for patients not eligible for transplant evolved quickly as stand-alone or "destination therapy" (DT) [6]. For patients with VADs who are noted to have myocardial recovery, either of these strategies can be converted to a "bridge to recovery" strategy, where dependence on the device is eventually weaned and subsequently explanted. The last indication, deemed "bridge to decision" (BTD), encompasses patients with potentially reversible physiologic or social contraindications to heart transplant. An individual patient's indication at the time of implantation commonly changes with ineligible patients transitioning to transplant and BTT patients forgoing heart transplantation or developing contraindications over time [7]. Currently, BTT and BTD are the indications for 21 and $33 \%$ of LVAD patients, whereas DT is the indication for $44 \%$, which is a significant increase representing a growing trend toward VAD placement for DT as devices have become more reliable $[8 \bullet$.

\section{Bridge to Transplant (BTT) and Bridge to Decision (BTD)}

VAD use as BTT offers many advantages over other bridging therapies. The VAD allows for patient mobility and participation in rehabilitation leading to increased survival compared to patients receiving inotrope therapy [9]. Further, patients ineligible for a heart transplant due to, what was previously considered, permanent end organ damage, such as fixed pulmonary hypertension, can experience recovery on LVAD support and subsequently undergo transplant. Such patients may experience similar transplant outcomes to those without the initial end organ damage [10]. This approach improves survival and quality of life for patients awaiting heart transplant while allowing better utilization of the limited number of donor hearts [11].

\section{Destination Therapy (DT)}

The landmark study describing VAD use in end-stage heart failure in transplant ineligible patients was the Randomized Evaluation of Mechanical Assistance for the Treatment of Congestive Heart Failure (REMATCH) study from 2001 [12]. This multicenter prospective randomized study compared patients who were deemed ineligible for heart transplant. One group of patients was randomized to VAD as DT using a first generation HeartMate pneumatically driven pulsatile device; the second group of patients was randomized to optimal medical management (OMM). The VAD group showed a $48 \%$ reduction in all-cause risk of death and an increased quality of life [12]. On the basis of the benefits demonstrated in REMATCH, this first generation LVAD received FDA approval for stand-alone therapy in transplant-ineligible patients and the DT indication was born.

Since this landmark trial, other studies have demonstrated the survival benefit of LVAD therapy compared to OMM for destination therapy in end-stage heart failure patients $[13,14]$. These later trials show sustained benefits in NYHA class, decreased stroke and infection rates and enhanced survival rates with recent studies reporting $77 \%$ 2-year survival [11]. With improvements in VAD technology as well as increased physician experience, improving complication profiles and better patient selection, the number of DT VADs continues to increase [15]. 
Bridge to Recovery (BTR)

Progressive myocardial remodeling in end-stage heart failure is traditionally thought to be largely irreversible, but recent evidence suggests this may not be completely true [16•]. Strategies consisting of ventricular unloading with a VAD coupled with aggressive medical therapy consisting of ACE-inhibitors, $\beta$-blockers, angiotensin receptor blockers and aldosterone antagonists attempt to induce reverse remodeling both molecularly and functionally, with some reports demonstrating encouraging results $[17 \cdot, 18$, 19]. The theoretical possibility of myocardial recovery with LVAD support has not been realized clinically, however. While clinical recovery is appealing, reported rates are low, and some initially observed improvements are short lived, most considerably in patients who had prolonged heart failure prior to VAD placement or those with a persistently dilated left ventricle [20, 21].

\section{Implantation (Fig. 1)}

The surgical technique for the left ventricular assist device (LVAD) implantation involves accessing the heart through a sternotomy, variably creating a pre-peritoneal pocket to house the pump and creating an anastomosis between the left ventricular apex and the inflow conduit and between the ascending aorta and the outflow graft [22]. Many LVADs, including the HeartMate II (Thoratec Corp., Pleasanton, CA), have an inflow conduit and outflow graft with a component of Dacron. The HeartWare HVAD

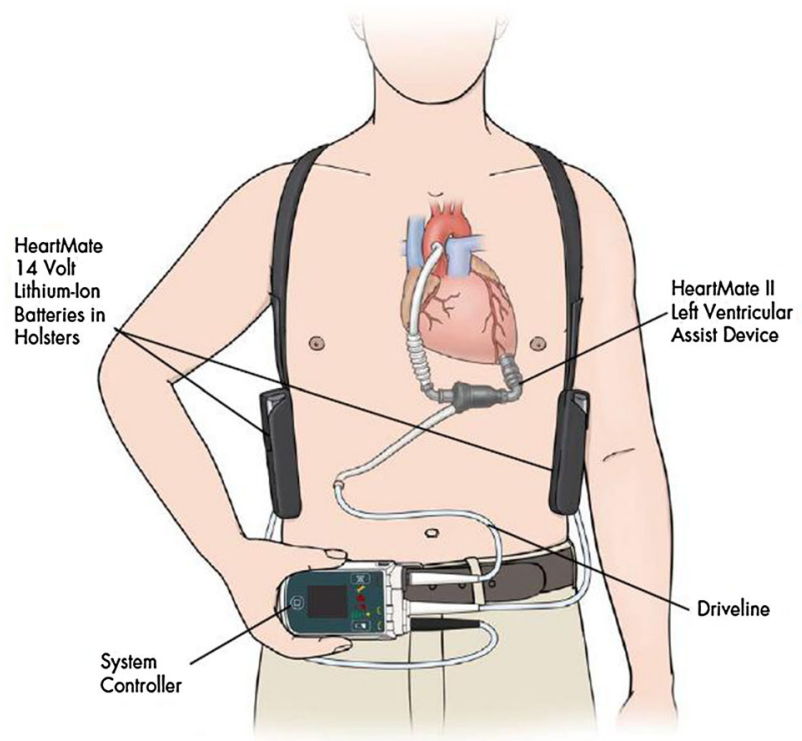

Fig. 1 Schematic diagram of a patient with the Thoratec HeartMate II LVAD. Reprinted with the permission from Thoratec Corp.
(Heartware, Inc., Framingham, MA) is positioned intrapericardially and connected to the inflow cannula directly, eliminating the need for a surgical pocket [23]. The Jarvik 2000 (Jarvik Heart, Inc., New York, NY) is positioned intraventricularly with an outflow cannula out of the left ventricle to the ascending aorta [24]. A driveline, which is tunneled out through the skin to a controller, provides power and controls pump speed. Less invasive sternal sparing surgical techniques have been described, ultimately intended to decrease morbidity for implantation, but their use is currently limited [25].

\section{Device Design and Evolution}

VADs have evolved over time from the initial pump of the 1960s. The first generation pumps were displacement, pulsatile pumps, mimicking the action of the heart. Currently, second generation devices employ continuous-flow technology, decreased pump size, more efficient energy use, remove the need for valves and reduce the number of moving parts to one. These changes give the advantage of longer battery life, decreased risk of infections, reduced hemolysis, reduced pump failure and increased durability [26]. The third generation devices, also continuous flow, have magnetically levitated noncontact bearings, which decrease friction and reduce heat generation, consequently continuing the increase in durability and efficiency [27].

The first generation devices showed a $48 \%$ reduction in the risk of death when compared to OMM [12]. Since this time, outcomes assessing the second and third generation continuous-flow devices against the first generation pulsatile devices have shown superiority of the second generation in improved survival with fewer adverse events and pump failures $[28,29,30 \bullet$. Additionally, these smaller devices are available for use in smaller patients [31]. Nearly all VADs placed currently in the adult population are continuous-flow devices [8•].

\section{Current Devices}

\section{HeartMate II (Fig. 2)}

The HeartMate II, a second generation VAD, utilizes an axial flow design to give a continuous flow of up to $10 \mathrm{l} /$ min [29]. It sits within a surgically created pocket, though it is small, with a displaced volume of $63 \mathrm{ml}$, which allows its utilization in smaller patients [28]. It was designed to have only one moving part to increase durability, with the bearing wear on explanted devices having been tested with an estimated life of $>27$ years [32]. It has been extensively studied and shows fewer adverse events and superior 
Fig. 2 a HeartMate II LVAD with attached inflow and outflow conduits. b Radiograph of implanted HeartMateII LVAD. Images reprinted with the permission of HeartWare International Inc.

\section{a}

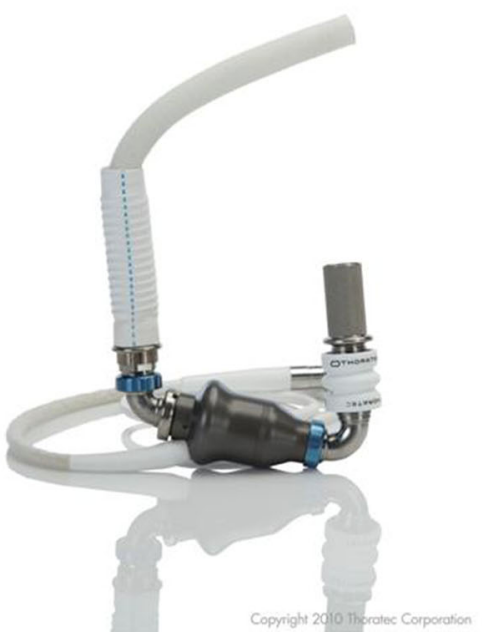

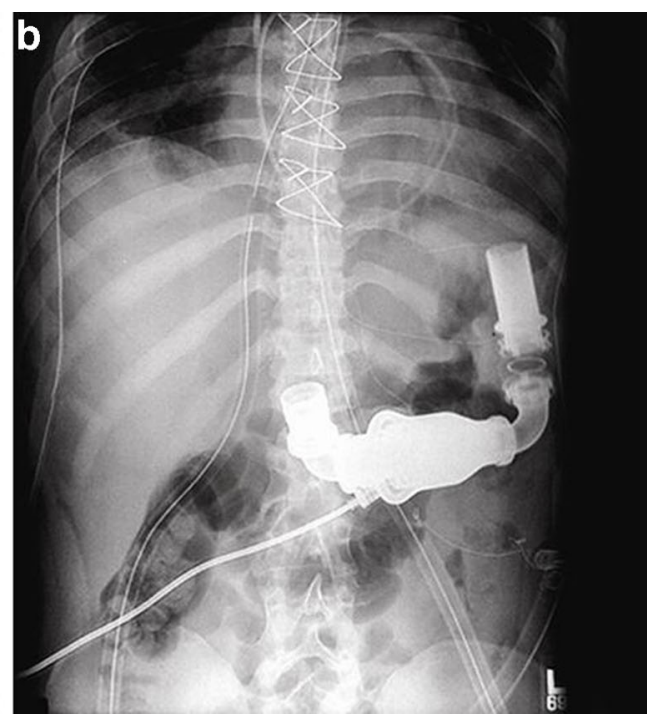

overall survival compared to the pulsatile devices for both BTT and DT with a 2-year survival of 68 versus $55 \%$ [22, 29].

\section{HeartWare HVAD (Fig. 3)}

HeartWare HVAD, a third generation VAD, has been validated by multiple studies showing safety and good clinical outcomes $[33,34,35 \bullet, 36]$. Its small size and centrifugal design allow for some unique advantages. Its primary advantage is its intrapericardial placement, avoiding the need for an abdominal or pre-peritoneal pocket [35•]. The small design, with a displaced volume of only $50 \mathrm{ml}$, also allows for placement in smaller patients [23]. The centrifugal design allows it to achieve a maximum flow of $10 \mathrm{l} / \mathrm{min}$ at substantially lower RPMs than the axial design [35•]. Recently, in the ADVANCE trial the HVAD was implanted for BTT with $86 \%$ 1-year survival establishing noninferiority for the BTT indication [34]. The ENDURANCE trial, a non-inferiority trial evaluating the HeartWare HVAD as long-term DT, finished enrolling 450 patients in May of 2012, with results pending [37].

\section{Other VADs}

Other VADs exist in various states of development and testing, some with unique advantages. The DuraHeart was the first magnetically levitated, noncontact pump and has shown safety and efficacy in European studies [38]. The Jarvik 2000 has a small design and sits in an intraventricular position, therefore eliminating the need to create a pump pocket. It also has the option of a driveline that can be anchored to the skull in an attempt to reduce infectious complications [24]. The LionHeart LVAD was a unique
LVAD system that employed a transcutaneous energy conduction system to allow full implantability. While this system eliminated driveline infectious risk, other complications remained or increased [39].

\section{Complications Associated with Ventricular Assist Devices}

Multiple potential complications can occur after VAD implantation and during ongoing support. Common postoperative complications include bleeding, sepsis and right heart failure. Despite improved surgical techniques and increased experience, bleeding remains a common and often devastating complication. Although perioperative bleeding rates have decreased with increased surgical experience, reoperation for bleeding still occurs at a rate of $15-30 \%[28,29,30 \bullet, 33,35 \bullet]$. Driveline infections were once seemingly unpreventable; however, the current incidence has decreased from $28 \%$ to as low as $14 \%$ in recent series [28, 33]. Thrombotic and bleeding complications persist with ongoing support. Embolic stroke is another potential complication of VADs. The incidence of stroke varies between studies and depends on pump characteristics and patient-related factors. Stroke generally ranges from 6 to $17 \%$ for continuous-flow VADs with slightly higher incidences reported with the HeartWare HVAD [28, $29,30 \bullet, 33,35 \bullet, 40 \bullet$. Mechanical complications such as pump failure have generally decreased as devices have improved with recent studies having shown a dramatic decrease in the number of primary pump failures to well below $5 \%[28,29,33,35 \cdot$. Recent studies, however, have noted a slight but real increase in early pump thrombosis, an observation without a clear etiology to date $[8 \bullet, 41]$. 
Fig. 3 a HeartWare HVAD device with ventricular sewing cuff. b Implanted HeartWare HVAD with inflow directly from the left ventricle and outflow to the ascending aorta. Images reprinted with the permission of HeartWare International Inc.

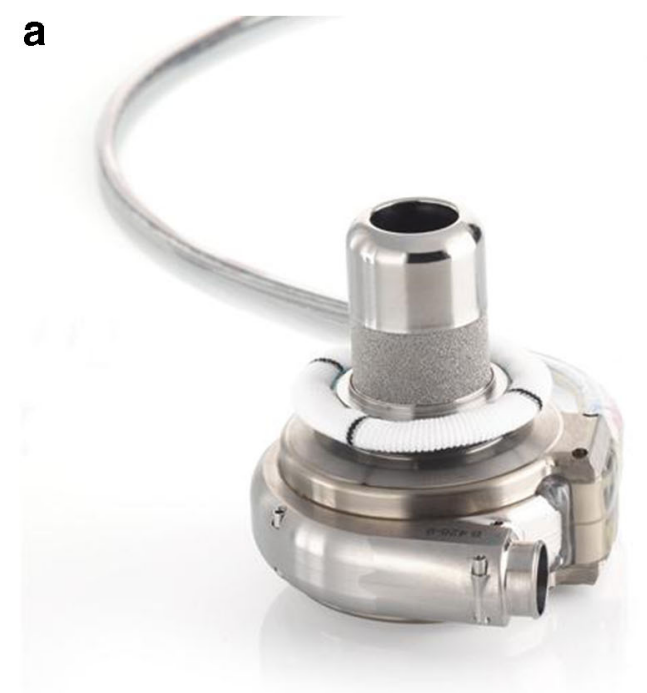

b

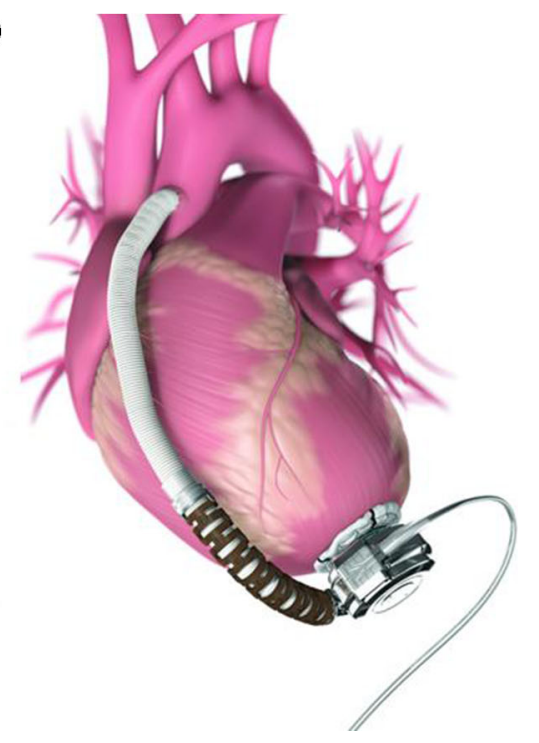

Right heart failure is another major postoperative and late complication associated with left ventricular unloading. While the mechanisms are not entirely understood, it has been hypothesized that the unloading of the left ventricle causes a shift of the interventricular septum that the right ventricle may be unable to overcome [42]. Development of this complication may necessitate the placement of a right ventricular assist device (RVAD) or contribute to progressive end organ dysfunction, such as renal failure. Right ventricular (RV) failure is divided into two groups based on the timing of inotropic support. If inotropic support is continuous since LVAD insertion or starts prior to 14 days postoperatively, it is considered early RV failure, and if it is initiated after 14 days postoperatively, it is considered late RV failure. Patients with early RV failure requiring either RVAD or inotropic therapy have been shown to have decreased 1-year survival when compared to patients without RV failure [43]. The incidence of right heart failure is decreased with more recent devices but is still considerable at 5-24\% [30•, 33, 43, 44].

Although the current VADs have shown increased survival with overall decreased adverse events, the diminished pulse pressure, inherent to the continuous-flow design, has been hypothesized as a cause of previously unforeseen complications [42]. This narrowed pulse pressure has been implicated as a cause of increased gastrointestinal bleeding episodes because of increased arteriovenous malformations and an acquired von Willebrand syndrome, mimicking Heyde's syndrome seen with aortic stenosis [34, 45]. Also, there is some evidence showing increased development of aortic insufficiency due to a constantly elevated transvalvular gradient, though this can be diminished by adjusting the VAD settings such that the aortic valve opens periodically $[42,46]$.

\section{Pediatrics}

The pediatric population presents unique challenges for a ventricular assist device. Many of the children who could benefit from VAD implantation are much smaller than adults; thus, their frame is not able to accommodate an adult-sized VAD. These smaller size patients require lower VAD flows achieved through a lower than normal operating rpm range with increased device thrombosis risk. Due to these technical and size challenges, the development of VADs for the pediatric population has lagged. Current VAD utilization in children with heart failure is becoming more common with the FDA approval of the Berlin device, a pneumatically driven pulsatile VAD [47].

The etiology of heart failure in the pediatric population differs from that of adults, as does the alternative treatments, such as ECMO, which are often employed successfully as a bridge to recovery [48]. Unfortunately, children ultimately in need of a heart transplant not uncommonly have substantial wait times [49]. The Berlin EXCOR has been shown to increase survival significantly when compared to the ECMO [47, 50]. With the new smaller second generation adult devices, implantation in larger teenagers has been possible, with the HeartMate II implanted in teenagers with a BSA $>1.5 \mathrm{~m}^{2}$ and the smaller HeartWare HVAD implanted in adolescents with BSA approaching $1.0 \mathrm{~m}^{2}$ with some modifications of the surgical technique [31].

\section{Ethical Concerns}

As the medical community continues to develop life-saving technology, ethical dilemmas frequently arise, and VADs 
are rarely considered in advanced directives [51]. As VAD placement is discussed with patient and family, end of life issues must be addressed, not only because of the seriousness of the current illness and the potential mortality of the operation but also because the treatment equates to mobile life support [52•]. While VADs have repeatedly been shown to increase quality of life, the possibility of a patient eventually asking to withdraw this life-prolonging care has been seen in multiple studies to be approximately $20 \%$ [51, 53]. While some believe that once implanted a VAD becomes one with the patient and deactivating it amounts to euthanasia, most ethicists agree that deactivation of the VAD equates to withdrawal of life support and the terminal insult is that of heart failure, not the deactivation of the VAD by the physician [54].

\section{VAD Underutilization}

VADs, as their outcomes continue to improve, are being utilized significantly more often [15]; however, there are certainly many patients who could benefit from a VAD who do not receive it. A recent study found that only 291 out of 297,866 patients who were candidates for a VAD received them [55]. Specific underserved populations have been identified, namely women and African Americans [56]. With the development of smaller VADs, the gap between use in men and women is improving [28]. The disparities in VAD utilization could be reduced with the education of physicians in the community and a decrease in the overall disparities in access to healthcare seen by minority races [55].

\section{Financial Impact}

When evaluating emerging therapies with broad applicability, cost must also be considered. Patients who undergo continuous-flow VAD implantation have significantly higher costs than patients who are medically managed ( $\$ 360,407$ vs. $\$ 62,856)$ but have an increase in quality-adjusted life years (QALY) (1.87 vs 0.37), giving an incremental cost-effectiveness ratio of $\$ 198,184$ per QALY [57 $]$. This is well above the commonly accepted threshold [58]; however, when considering the incremental cost-effectiveness ratio during the REMATCH era of $\$ 802,700$ [59], this represents tremendous strides toward reaching the cost- effective benchmark. This trend has been driven by improving patient survival with less frequent complications and hospitalizations and reduced failure requiring device replacement [57•]. One should only expect the trend of a reduction in the incremental costeffectiveness ratio to continue with improving devices and improved patient selection.

\section{Future Progress}

Improving patient outcomes with the further evolution of mechanical circulatory support remains essential to the increased utilization of LVADs in patients with heart failure. Ongoing device development, with increasing use in less sick patients and lower cost of care, will continue to drive growth of mechanical circulatory support. Currently, there are multiple potentially modifiable factors to improve outcomes with patient selection being a major determinant of outcome. Survival at 3 years has been shown to vary from 95.8 to $51.1 \%$ based on the INTERMACS profile $[52,60]$. Initially implantation of LVADs was reserved for only the sickest patients [15]. As outcomes have improved, a downshifting of risk has been realized such that VADs are being placed in less critically ill patients, leading to improved outcomes [5]. Recent data show the number of patients "crashing onto VAD" is decreasing [5, 15]. Prognostically, the destination therapy risk score was devised for risk stratification, though it was originally developed from pulsatile device use (Table 2) [7, 61•]. Newer risk scores such as the HeartMate II Risk Score are in general agreement and report increasing mortality risk in patients with increasing age, renal dysfunction, hypoalbuminemia, coagulopathy and those receiving LVAD support at less experienced centers [62•].

Currently, two ongoing trials, the REVIVE-IT and ROADMAP trials, are investigating LVAD implantation in less ill patients earlier in heart failure treatment [27]. Both trials will evaluate placement of the HeartMate II VAD prior to inotrope dependency and other organ dysfunction in an attempt to further prolong survival and increase quality of life [63].

In the current era of mechanical circulatory support, treatment decisions may be based on trials performed with older generation LVADs. Incremental innovation may

Table 2 Risk factors for 90-day mortality for DT with weighted score

\begin{tabular}{ll}
\hline Risk factor & Score \\
\hline Platelet count $\leq 148 \times 10^{3} / \mu \mathrm{l}$ & 7 \\
Serum albumin $\leq 3.3 \mathrm{~g} / \mathrm{dl}$ & 5 \\
International normalization ratio $>1.1$ & 4 \\
Vasodilator therapy & 4 \\
Mean pulmonary artery pressure $\leq 25 \mathrm{mmHg}$ & 3 \\
Aspartate aminotransferase $>45 \mathrm{U} / \mathrm{ml}$ & 2 \\
Hematocrit $\leq 34 \%$ & 2 \\
Blood urea nitrogen $>51 \mathrm{Y} / \mathrm{dl}$ & 2 \\
No intravenous inotropes & 2 \\
\hline
\end{tabular}

Low $=0-8$, medium $=9-16$, high $=17-19$, very high $>19$. Adapted from Leitz et al. [7] 

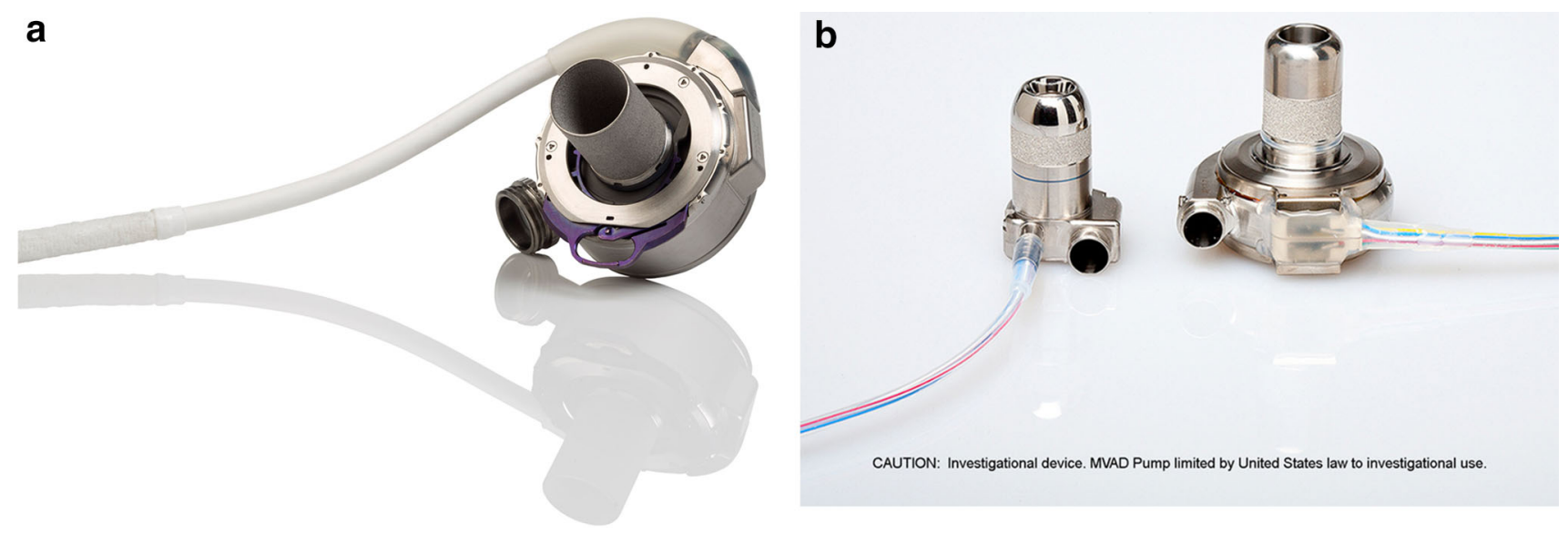

Fig. 4 Future devices currently under investigation, neither available for clinical use. a HeartMate III LVAD; b HeartWare MVAD. Images reprinted with permission from HeartWare International, Inc., and Thoratec Corp.

utilize hybrid development strategies such as employing fully implantable systems in currently approved devices before employing these innovations in future devices.

The HeartMate III (Fig. 4a) is an investigational device featuring a continuous-flow centrifugal design with a fully magnetically levitated noncontact rotor [64]. Additionally, an induced pulse mode will be available to increase pulsatility with continuous flow assistance $[65,66]$. The surfaces will be textured to promote pseudoneointimal growth and may require aspirin only for anticoagulation [65]. In vivo testing in calves demonstrated good performance with normal end-organ function and the ability to produce physiological pulse pressure $[65,67]$.

Currently under development, the HeartWare MVAD (Fig. 4b) is a small axial flow device capable of delivering flows of $1-71 / \mathrm{min}$. One version of this model is being designed for intraventricular placement via a transapical approach with the outflow cannula positioned across the aortic valve [66]. It is smaller than the HVAD, with a displacement volume of only $22 \mathrm{ml}$, and has already been studied in an ovine model demonstrating safety and reliability over 90 days [68].

Synergy (CircuLite, Teaneck, NJ), a miniature pump implanted through a small thoracotomy representing the first wave of micro LVADS, is capable of providing partial circulatory support and received CE Mark approval in Europe in September 2012. Future refinements of this pump platform include the possibility of endovascular deployment [69].

\section{Conclusions}

Survival and complication rates for current LVAD use have been substantially improved over prior devices.
Newer devices, better patient selection and improved surgical technique and postoperative care have led to an increase in survival and a decrease in adverse events. While the device-related cost remains a concern, the cost of care continues to improve. Future innovations will focus on fully implantable systems, improved biocompatibility and less invasive implantation to drive lower long-term complication rates and drive further utilization.

\section{Compliance with Ethics Guidelines}

Conflict of Interest This work is supported in part by National Institutes of Health grant T32 HL007776. Scott C. Silvestry has served as a consultant for Thoratec and HeartWare, has received grant support from Thoratec, has received honoraria from Abiomed and has had travel/accommodations expenses covered/reimbursed by Thoratec. Jacob R. Miller and Christopher P. Lawrance declare that they have no conflict of interest.

Human and Animal Rights and Informed Consent This article does not contain any studies with human or animal subjects performed by any of the authors.

\section{References}

Recently published papers of particular interest have been highlighted as:

- Of importance

1. Go AS, et al. Heart disease and stroke statistics-2013 update: a report from the American Heart Association. Circulation. 2013;127(1):e6-245.

2. Krumholz HM, et al. Patterns of hospital performance in acute myocardial infarction and heart failure 30-day mortality and readmission. Circ Cardiovasc Qual Outcomes. 2009;2(5):407-13.

3. Yancy $\mathrm{CW}$, et al. ACCF/AHA guideline for the management of heart failure: executive summary: a report of the American 
College of Cardiology Foundation/American Heart Association Task Force on Practice Guidelines. Circulation. 2013;128(16): 1810-52.

4. Taylor DO, et al. Registry of the International Society for Heart and Lung Transplantation: Twenty-sixth Official Adult Heart Transplant Report-2009. J Heart Lung Transplant. 2009;28(10): 1007-22.

5. Stevenson LW, et al. INTERMACS profiles of advanced heart failure: the current picture. J Heart Lung Transplant. 2009;28(6):535-41.

6. Rose EA, et al. The REMATCH trial: rationale, design, and end points. Randomized evaluation of mechanical assistance for the treatment of congestive heart failure. Ann Thorac Surg. 1999;67(3): 723-30.

7. Lietz K, et al. Outcomes of left ventricular assist device implantation as destination therapy in the post-REMATCH era: implications for patient selection. Circulation. 2007;116(5): 497-505.

8. - Kirklin JK, et al. Fifth INTERMACS annual report: risk factor analysis from more than 6,000 mechanical circulatory support patients. J Heart Lung Transplant. 2013;32(2):141-56. Annual INTERMACS report detailing first 66 years of VAD data with trends of VAD usage, survival, risk factors, adverse events and quality of life data.

9. Aaronson KD, et al. Left ventricular assist device therapy improves utilization of donor hearts. J Am Coll Cardiol. 2002; 39(8):1247-54.

10. Zimpfer D, et al. Post-transplant survival after lowering fixed pulmonary hypertension using left ventricular assist devices. Eur J Cardiothorac Surg. 2007;31(4):698-702.

11. Long JW, et al. Improving outcomes with long-term "destination" therapy using left ventricular assist devices. J Thorac Cardiovasc Surg. 2008;135(6):1353-60 discussion 1360-1.

12. Rose EA, et al. Long-term use of a left ventricular assist device for end-stage heart failure. N Engl J Med. 2001;345(20):1435-43.

13. Stevenson LW, et al. Left ventricular assist device as destination for patients undergoing intravenous inotropic therapy: a subset analysis from REMATCH (Randomized Evaluation of Mechanical Assistance in Treatment of Chronic Heart Failure). Circulation. 2004;110(8):975-81.

14. Lietz K, Miller LW. Destination therapy: current results and future promise. Semin Thorac Cardiovasc Surg. 2008;20(3): 225-33.

15. Kirklin JK, et al. The Fourth INTERMACS Annual Report: 4,000 implants and counting. J Heart Lung Transplant. 2012;31(2):117-26.

16. - Birks EJ, Molecular changes after left ventricular assist device support for heart failure. Circ Res. 2013;113(6):777-91. An article showing the molecular, not necessarily functional, reversal of remodeling seen with heart failure.

17. - Morgan, J.A., et al., Left ventricular reverse remodeling with a continuous flow left ventricular assist device measured by left ventricular end-diastolic dimensions and severity of mitral regurgitation. ASAIO J. 2012;58(6):574-7. Evaluates continuous-flow devices, showing their ability to effectively unload the left ventricle and possibly lead to myocardial recovery.

18. Lamarche Y, et al. Successful weaning and explantation of the Heartmate II left ventricular assist device. Can J Cardiol. 2011;27(3):358-62.

19. Birks EJ, et al. Left ventricular assist device and drug therapy for the reversal of heart failure. N Engl J Med. 2006;355(18):1873-84.

20. Liang $\mathrm{H}$, et al. Prediction of cardiac function after weaning from ventricular assist devices. J Thorac Cardiovasc Surg. 2005;130(6): 1555-60.

21. Simon MA, et al. Left ventricular remodeling and myocardial recovery on mechanical circulatory support. J Card Fail. 2010; 16(2):99-105.
22. Struber M, et al. HeartMate II left ventricular assist device; early European experience. Eur J Cardiothorac Surg. 2008;34(2): 289-94.

23. Larose JA, et al. Design concepts and principle of operation of the HeartWare ventricular assist system. ASAIO J. 2010;56(4): 285-9.

24. Holman WL, et al. Device related infections: are we making progress? J Card Surg. 2010;25(4):478-83.

25. Gregoric ID, et al. A less invasive approach to axial flow pump insertion. J Heart Lung Transplant. 2008;27(4):423-6.

26. Givertz MM. Cardiology patient pages: ventricular assist devices: important information for patients and families. Circulation. 2011;124(12):e305-11.

27. Rodriguez LE, et al. Ventricular assist devices (VAD) therapy: new technology, new hope? Methodist Debakey Cardiovasc J. 2013;9(1):32-7.

28. Miller LW, et al. Use of a continuous-flow device in patients awaiting heart transplantation. N Engl J Med. 2007;357(9): 885-96.

29. Slaughter MS, et al. Advanced heart failure treated with continuous-flow left ventricular assist device. N Engl J Med. 2009; 361(23):2241-51.

30. - Park SJ, et al. Outcomes in advanced heart failure patients with left ventricular assist devices for destination therapy. Circ Heart Fail. 2012;5(2):241-8. Evaluates the HeartMate II VAD as DT comparing outcomes for patients operated on more recently, illustrating a significant decrease in adverse events and a trend toward improved survival with increased surgical experience and improved postoperative care.

31. Owens WR, et al. Initial clinical experience with the HeartMate II ventricular assist system in a pediatric institution. Artif Organs. 2010;34(7):600-3.

32. Sundareswaran KS, et al. Low bearing wear in explanted HeartMate II left ventricular assist devices after chronic clinical support. ASAIO J. 2013;59(1):41-5.

33. Popov AF, et al. Clinical experience with HeartWare left ventricular assist device in patients with end-stage heart failure. Ann Thorac Surg. 2012;93(3):810-5.

34. Aaronson KD, et al. Use of an intrapericardial, continuous-flow, centrifugal pump in patients awaiting heart transplantation. Circulation. 2012;125(25):3191-200.

35. - Strueber M, et al. Multicenter evaluation of an intrapericardial left ventricular assist system. J Am Coll Cardiol. 2011; 57(12):1375-82. Evaluate the HeartWare in multicenter single arm trial showing survival and quality of life data.

36. Wieselthaler GM, et al. Initial clinical experience with a novel left ventricular assist device with a magnetically levitated rotor in a multi-institutional trial. J Heart Lung Transplant. 2010; 29(11):1218-25.

37. Pagani FD, Rogers JG, et al. US ENDURANCE Destination therapy trial. 2013.

38. Morshuis M, et al. European experience of DuraHeart magnetically levitated centrifugal left ventricular assist system. Eur J Cardiothorac Surg. 2009;35(6):1020-7 discussion 1027-8.

39. Pae WE, et al. Does total implantability reduce infection with the use of a left ventricular assist device? The LionHeart experience in Europe. J Heart Lung Transplant. 2007;26(3):219-29.

40. $\bullet$ Lalonde SD, et al. Clinical differences between continuous flow ventricular assist devices: a comparison between HeartMate II and HeartWare HVAD. J Card Surg. 2013;28(5):604-10. Trial comparing HeartMate II and HeartWare HVAD showing similar survival with higher incidence of stroke and gastrointestinal bleeding in the HeartWare arm.

41. Starling RC, et al. Unexpected abrupt increase in left ventricular assist device thrombosis. N Engl J Med. 2014;370(1):33-40. 
42. Soucy KG, et al. Rotary pumps and diminished pulsatility: do we need a pulse? ASAIO J. 2013;59(4):355-66.

43. Kormos RL, et al. Right ventricular failure in patients with the HeartMate II continuous-flow left ventricular assist device: incidence, risk factors, and effect on outcomes. J Thorac Cardiovasc Surg. 2010;139(5):1316-24.

44. Lee S, et al. Effects of the HeartMate II continuous-flow left ventricular assist device on right ventricular function. J Heart Lung Transplant. 2010;29(2):209-15.

45. Crow S, et al. Acquired von Willebrand syndrome in continuousflow ventricular assist device recipients. Ann Thorac Surg. 2010;90(4):1263-9 discussion 1269.

46. Pak SW, et al. Prevalence of de novo aortic insufficiency during long-term support with left ventricular assist devices. J Heart Lung Transplant. 2010;29(10):1172-6.

47. Morales DL, et al. Bridging children of all sizes to cardiac transplantation: the initial multicenter North American experience with the Berlin Heart EXCOR ventricular assist device. J Heart Lung Transplant. 2011;30(1):1-8.

48. Ihnat CL, et al. Left ventricular assist device support as a bridge to recovery in young children. Congenit Heart Dis. 2011;6(3): 234-40.

49. Kirk R, et al. The Registry of the International Society for Heart and Lung Transplantation: thirteenth official pediatric heart transplantation report-2010. J Heart Lung Transplant. 2010; 29(10):1119-28.

50. Fraser CD Jr, et al. Prospective trial of a pediatric ventricular assist device. N Engl J Med. 2012;367(6):532-41.

51. Mueller PS, et al. Ethical analysis of withdrawing ventricular assist device support. Mayo Clin Proc. 2010;85(9):791-7.

52. - Boyle AJ, et al. Clinical outcomes for continuous-flow left ventricular assist device patients stratified by pre-operative INTERMACS classification. J Heart Lung Transplant. 2011;30(4): 402-7. A study of 101 patients analyzing outcomes based on INTERMACS profile at the time of VAD implantation, illustrates significant difference in outcomes.

53. MacIver J, Ross HJ. Withdrawal of ventricular assist device support. J Palliat Care. 2005;21(3):151-6.

54. Bruce CR, Brody B, Majumder MA. Ethical dilemmas surrounding the use of ventricular assist devices in supporting patients with end-stage organ dysfunction. Methodist Debakey Cardiovasc J. 2013;9(1):11-4.

55. Joyce DL, et al. Disparities in access to left ventricular assist device therapy. J Surg Res. 2009;152(1):111-7.
56. Miller LW. Left ventricular assist devices are underutilized. Circulation. 2011;123(14):1552-8 discussion 1558.

57. - Rogers JG, et al. Cost-effectiveness analysis of continuous-flow left ventricular assist devices as destination therapy. Circ Heart Fail. 2012;5(1):10-6. Overview of cost-effectiveness, showing a high but improving cost of care.

58. Miller LW, Guglin M, Rogers J. Cost of ventricular assist devices: can we afford the progress? Circulation. 2013;127(6):743-8.

59. Loffredo CA, et al. Prevalence of congenital cardiovascular malformations among relatives of infants with hypoplastic left heart, coarctation of the aorta, and d-transposition of the great arteries. Am J Med Genet A. 2004;124A(3):225-30.

60. Bhama JK, et al. Impact of abdominal complications on outcome after mechanical circulatory support. Ann Thorac Surg. 2010; 89(2):522-8 discussion 528-9.

61. - Slaughter MS, Meyer AL, Birks EJ. Destination therapy with left ventricular assist devices: patient selection and outcomes. Curr Opin Cardiol. 2011;26(3):232-6 Analysis showing improved outcomes based on patient selection.

62. ${ }^{-}$Cowger J, et al. Predicting survival in patients receiving continuous flow left ventricular assist devices: the HeartMate II risk score. J Am Coll Cardiol. 2013;61(3):313-21. Illustrates risk factors that can be used prognostically prior to VAD implantation.

63. Baldwin JT, Mann DL. NHLBI's program for VAD therapy for moderately advanced heart failure: the REVIVE-IT pilot trial. J Card Fail. 2010;16(11):855-8.

64. Bourque $\mathrm{K}$, et al. HeartMate III: pump design for a centrifugal LVAD with a magnetically levitated rotor. ASAIO J. 2001;47(4): 401-5.

65. Farrar DJ, et al. Design features, developmental status, and experimental results with the Heartmate III centrifugal left ventricular assist system with a magnetically levitated rotor. ASAIO J. 2007;53(3):310-5.

66. Molina EJ, Boyce SW. Current status of left ventricular assist device technology. Semin Thorac Cardiovasc Surg. 2013;25(1): $56-63$.

67. Loree HM, et al. The Heartmate III: design and in vivo studies of a maglev centrifugal left ventricular assist device. Artif Organs. 2001;25(5):386-91.

68. McGee E, Jr. et al. In Vivo Evaluation of the HeartWare MVAD Pump. J Heart Lung Transplant. 2013.

69. Circulite, Technology Overview. 2013. 